ISSN: 2224-0616

Int. J . Agril. Res. Innov. \& Tech. 4 (2): 35-40, December, 2014 Available online at http:// www.ijarit.webs.com

\title{
IMPROVEMENT OF POTATO BASED CROPPING PATTERNS BY INCLUSION OF SHORT DURATION MUNGBEAN AND T. AMAN RICE IN MONGA PRONE AREAS OF RANGPUR
}

\author{
M.K. Islam1, M.S. Mahfuz ${ }^{2}$, S. Ghosh ${ }^{3}$, A.S.M.Y. Ali² and M.Z. Hasnat ${ }^{4}$ \\ Received 10 May 2014, Revised 28 October 2014, Accepted 22 December 2014, Published online 31 December 2014
}

\begin{abstract}
The experiment was carried out to compare the improved cropping patterns against the farmers existing potato based cropping patterns having no mungbean/brown manure crop for higher yield, economic return and income generation in agricultural field in the off period following RCBD design with three replications at farmer's field at Paikan Gangachara, Rangpur district during SeptemberOctober. The treatments (cropping patterns) were $\mathrm{T}_{1}=\mathrm{T}$. aman rice (BINA 7) - Potato - Mungbean (BARI mungbean 6) (Improved pattern), $\mathrm{T}_{2}=\mathrm{T}$. aman rice (BR11) - Potato - Fallow (Farmers pattern), $\mathrm{T}_{3}=\mathrm{T}$. aman rice (BINA 7) - Potato + Maize intercrop - Mungbean (Improved pattern) and $\mathrm{T}_{4}=\mathrm{T}$. aman rice (BR11) - Potato / Maize relay (Farmers pattern). The highest yield (4.16 t ha-1) was recorded in $\mathrm{T}_{2}$ (BR11) which is statistically at par with $\mathrm{T}_{4}\left(4.15 \mathrm{t} \mathrm{ha}^{-1}\right)$ but higher than the other treatments. Early planting sole potato $\left(\mathrm{T}_{1}\right)$ gave highest yield $\left(26.10 \mathrm{t} \mathrm{ha}^{-1}\right)$ which was significantly higher than all other treatments. Late planting sole potato $\left(T_{2}\right)$, intercropped early potato $\left(T_{3}\right)$ and relay potato $\left(\mathrm{T}_{4}\right)$ showed similar yield $\left(23.61-24.79 \mathrm{t} \mathrm{ha}^{-1}\right)$. Intercropped $\left(\mathrm{T}_{3}\right)$ and Relay $\left(\mathrm{T}_{4}\right)$ maize did not vary significantly in the studied parameters and yields were 8.21 and $7.92 \mathrm{t} \mathrm{ha}^{-1}$, respectively. Mungbean after sole potato $\left(\mathrm{T}_{1}\right)$ gave higher number of pods/plant (17.25), and yield (1.47 t ha-1) which is significantly higher than those of $\mathrm{T}_{3}$ (14.89 and $1.28 \mathrm{t}$ ha-1, respectively). Highest gross return (GR) (Tk. 417720) and gross margin (GM) (Tk. 220220) were calculated in improved pattern $\mathrm{T}_{3}$ and the lowest of those (Tk. 289670, Tk. 146020) in farmers pattern $T_{2}$. The other improved pattern $T_{3}$ was the second highest performer considering GR and GM. But BCR (2.21) was highest in $\mathrm{T}_{1}$ and second highest in $T_{3}$. The results indicated that the improved patterns $\left(T_{1}, T_{3}\right)$ were better than farmers pattern $\left(T_{2}, T_{4}\right)$. The improved pattern $\left(T_{1}\right)$ gave GR Tk. 67890 and GM Tk. 51785 higher than farmers pattern $\left(\mathrm{T}_{2}\right)$. Similarly, the other improved pattern $\left(\mathrm{T}_{3}\right)$ showed Tk. 51870 and Tk. 37395 higher than farmers pattern $\left(\mathrm{T}_{4}\right)$. The improved pattern $\mathrm{T}_{1}$ and $\mathrm{T}_{3}$ created 45 working day job for the labour for harvesting early matured rice in the Monga/ jobless period (October) while farmers' pattern gives only 3 days work. The mungbean included improved cropping patterns can be suggested for increased production, economic return and Monga mitigation (work opportunity in off period) in Rangpur.
\end{abstract}

Keywords: Improvement, Pattern, Potato, Maize, Mungbean, Monga, Labour

\footnotetext{
${ }^{1}$ Senior Scientific Officer, Bangladesh Agricultural Research Institute, Burirhat, Rangpur, Bangladesh

${ }^{2}$ Regional Farm Broadcasting Officer, Agriculture Information Service, Rangpur, Bangladesh

${ }^{3}$ Scientific Officer, Bangladesh Agricultural Research Institute, Burirhat, Rangpur, Bangladesh

${ }^{4}$ Information Officer, Agriculture Information Service, Dhaka, Bangladesh

*Corresponding author's email: shohag_agri@yahoo.com (M.S. Mahfuz)
}

\section{Introduction}

Cultivable land of Bangladesh is reducing day by day because of new industries and human settlement. On the contrary, demand for food is increasing due to population pressure. Crop production has to be increased to feed the everincreasing mouths. Gangachara is one of the Monga (seasonal unemployment) prone areas of Rangpur. A huge area is under potato cultivation in this area and farmers are practicing different potato based cropping patterns like T. aman rice - Potato - Fallow, T. aman rice - Potato/ Maize, T. aman rice - Potato - Jute etc. They use long duration $\mathrm{T}$. aman variety BR 11 causing delay in potato planting in December, which ultimately reduces yield and favours crop failure due to late blight disease. November is best for potato planting which can avert the disease by maturing crop before the severe infestation period. In these patterns, there is no work for the poor in October - November in agricultural field in Rangpur region. Poor people starve at that time which is called Monga in local dialect and government has to take extra measure to feed them. Short duration T. Aman rice (BINA Dhan 7) can facilitate timely/early potato planting (November) which is important for good yield. It 
also can create job opportunity for the poor in Monga period (September-October) for harvesting rice. On the other hand, a good soil should have organic matter content more than 3.5 per cent, while more than $60 \%$ of our cultivated soil contains organic matter at low level $(<1.7 \%)$. Recycling of organic matter is essential for maintaining soil fertility (BARC, 2001). Declining soil fertility is a major reason for lower crop yield in Bangladesh (Chowdhury et al., 2003). Inclusion of green manure crop in the cropping pattern is a solution to soil degradation. Adjustments in cropping patterns are necessary on a large scale incorporating green manuring and grain legume crops to improve soil health (BARC, 2001). If maize is intercropped with potato, it can facilitate two months time to grow mungbean before T. Aman rice. Intercropping system maximizes complementary use of growth resources (Krishna and Raikhelkar, 1997) and enhances the total productively (Umrani et al., 1984). Potato single stand requires high levels of inputs, which are in most instances beyond the economic capacity of farmers (Luis and Manrique, 1996). Moreover, it improves soil health by reducing pathogenic microorganisms survive in soil by creating disruption of life cycle (Dey, 2001). Under some conditions, intercropping can usefully contribute to the control of pest or disease populations and the reduction of yield loss (Trenbath, 1993). As the field duration of the variety BARI Mungbean 6 is short ( 55 days) and pods mature almost at a time,

Table 1. Planting and harvesting time of crops after harvesting of pod biomass incorporation into soil can help enrich soil by adding organic matter. A huge foreign currency can also be saved for importing pulse from abroad. Keeping the views in mind the experiment was undertaken to accommodate mungbean into farmers cropping pattern for increased yield and economic return, to generate work in agricultural field in the off period during September-October and to increase pulse production and save foreign currency for import.

\section{Methodology}

The experiment was conducted at farmer's field at village Paikan under Gangachara Upozilla of Rangpur district following RCBD design with three replications. Twenty-five decimal lands for each pattern were selected. The improved cropping patterns were compared with the farmers existing potato based patterns having no mungbean/ brown manure crop. The treatments (cropping patterns) were $-\mathrm{T}_{1}=\mathrm{T}$. aman rice (BINA 7) - Potato - Mungbean (BARI mungbean 6) (Improved pattern), $\mathrm{T}_{2}=\mathrm{T}$. aman rice (BR11) - Potato - Fallow (Farmers pattern), $\mathrm{T}_{3}=\mathrm{T}$. aman rice (BINA 7) - Potato + Maize intercrop Mungbean (Improved pattern) and $\mathrm{T}_{4}=\mathrm{T}$. aman rice (BR11) - Potato / Maize relay (Farmers pattern). The patterns initiated in July 2011 and planting and harvesting time of crops are given below in the Table 1.

\begin{tabular}{|c|c|c|c|c|}
\hline $\mathrm{T}_{1}$ & T. aman rice (BINA 7) & Potato & Mungbean & - \\
\hline & 21 July - 21 Oct & 05 Nov - 06 Feb & $21 \mathrm{Feb}-21 \mathrm{Apr}$ & \\
\hline $\mathrm{T}_{2}$ & $\begin{array}{l}\text { T. aman rice (BR11) } \\
05 \text { Auqust - } 05 \text { Dec }\end{array}$ & $\begin{array}{l}\text { Potato } \\
10 \text { Dec - } 03 \text { Mar }\end{array}$ & Fallow & - \\
\hline $\mathrm{T}$ & T. aman rice (BINA 7) & Potato & Maize & Mungbean \\
\hline & 21July - 21 Oct & $5 \mathrm{Nov}-20 \mathrm{Jan}$ & $13 \mathrm{Nov}-12 \mathrm{Apr}$ & 6 Apr - 02 J un \\
\hline $\mathrm{T}_{4}$ & T. aman rice (BR11) & Potato & Maize & \\
\hline & 05 August - 05 Dec & $10 \mathrm{Dec}-01 \mathrm{Mar}$ & 01Feb- 04Jun & \\
\hline
\end{tabular}

After harvesting, mungbean biomass (brown manure) was incorporated into soil to improve and maintain soil fertility. Recommended doses of fertilizer and management practice were maintained. Intercropping maize was sown in between potato rows at 7 days after sowing of potato. Relay maize was sown on the side of potato ridge at 50 days after sowing of potato. Plant spacing was $60 \mathrm{~cm} \times 25 \mathrm{~cm}$ for potato and intercrops and relay maize, $75 \mathrm{~cm} \times 20 \mathrm{~cm}$ for sole maize, $30 \mathrm{~cm} \times 10 \mathrm{~cm}$ for mungbean and 20 $\times 15 \mathrm{~cm}$ for T. aman rice. Data on yield and yield components was taken and data were analyzed statistically following Gomez and Gomez (1984) and mean separation was done by LSD. Economic analysis was also done. The total benefits of the patterns were calculated.

\section{Results and Discussion}

\section{T. Aman rice}

Plant height, number of effective tiller/ hill, 1000grain weight and yield increased significantly due to treatments (Table 2). The BR11 rice produced significantly higher plant height $\left(106.2 \mathrm{~cm}\right.$ in $\mathrm{T}_{4}$ and $106.0 \mathrm{~cm}$ in $\left.\mathrm{T}_{2}\right)$ than BINA dhan $7(99.07 \mathrm{~cm}$ in $\mathrm{T}_{3}$ and $98.90 \mathrm{~cm}$ in $\mathrm{T}_{1}$ ). Similar trend was observed in other parameters, which differed significantly due to treatment effect. The highest yield (4.16 t ha-1) was recorded in $T_{2}$ which is statistically at par with $\mathrm{T}_{4}\left(4.15 \mathrm{t} \mathrm{ha}^{-1}\right)$ but significantly higher than the other treatments $\mathrm{T}_{1}$ (3.98 tha-1) and $\mathrm{T}_{3}\left(3.96 \mathrm{t} \mathrm{ha}^{-1}\right)$. Treatment $\mathrm{T}_{1}$ and $\mathrm{T}_{3}$ is similar in producing yield. Higher number of effective tiller and 1000-grain weight favours higher yield in BR11. 
Table 2. Yield and yield contributing characters of T. aman rice in farmer's field, Rangpur

\begin{tabular}{lllllll}
\hline $\begin{array}{l}\text { Treatments } \\
\text { (Cropping patterns) }\end{array}$ & $\begin{array}{l}\text { Plant } \\
\text { height }(\mathrm{cm})\end{array}$ & $\begin{array}{l}\text { Effective } \\
\text { tiller/ hill } \\
\text { (no) }\end{array}$ & $\begin{array}{l}\text { Panicle } \\
\text { length }(\mathrm{cm})\end{array}$ & $\begin{array}{l}\text { Grains/ } \\
\text { panicle } \\
\text { (no) }\end{array}$ & $\begin{array}{l}1000 \\
\text { grain } \\
\text { weight } \\
\text { (g) }\end{array}$ & $\begin{array}{l}\text { Yield } \\
\left(\mathrm{t} \mathrm{ha}^{-1}\right)\end{array}$ \\
\hline $\mathrm{T}_{1}=$ BINA7-Pot-Mung & $98.90 \mathrm{~b}$ & $8.40 \mathrm{~b}$ & 23.60 & 69.00 & $22.17 \mathrm{~b}$ & $3.98 \mathrm{~b}$ \\
$\mathrm{~T}_{2}=$ BR11-Pot-Fallow & $106.0 \mathrm{a}$ & $8.73 \mathrm{a}$ & 23.30 & 69.40 & $24.63 \mathrm{a}$ & $4.16 \mathrm{a}$ \\
$\mathrm{T}_{3}=$ BINA7-P+M-Mung & $99.07 \mathrm{~b}$ & $8.46 \mathrm{~b}$ & 23.77 & 67.00 & $21.97 \mathrm{~b}$ & $3.96 \mathrm{~b}$ \\
$\mathrm{~T}_{4}=$ BR11-Pot/Maize & $106.2 \mathrm{a}$ & $8.50 \mathrm{ab}$ & 23.97 & 69.67 & $25.13 \mathrm{a}$ & $4.15 \mathrm{a}$ \\
\hline $\mathrm{CV}(\%)$ & 3.2 & 1.5 & 1.77 & 2.33 & 4.17 & 2.12 \\
\hline Level of Significance & $*$ & $*$ & $\mathrm{NS}$ & $\mathrm{NS}$ & $*$ & $*$ \\
\hline LSD $(0.05)$ & 6.56 & 0.26 & & & 1.96 & 0.17 \\
\hline
\end{tabular}

*Significant at 0.05 level of significance

Letters in a column having similar or no letter did not differ significantly

\section{Potato}

All the parameters except number of stem/ hill varied significantly (Table 3). Early planting sole potato $\left(\mathrm{T}_{1}\right)$ gave highest yield $\left(26.10 \mathrm{t} \mathrm{ha}^{-1}\right)$ which was significantly higher than all other treatments. Late planting sole potato $\left(\mathrm{T}_{2}\right)$, intercropped early potato $\left(\mathrm{T}_{3}\right)$ and relay potato $\left(\mathrm{T}_{4}\right)$ showed similar yield (23.61 - $\left.24.79 \mathrm{t} \mathrm{ha}^{-1}\right)$. Highest plant height $(68.97 \mathrm{~cm})$, and number (8.76) and weight (466.7 g) of tuber/ hill contributed to the highest yield in $\mathrm{T}_{1}$. Treatment $\mathrm{T}_{2}, \mathrm{~T}_{3}$ and $\mathrm{T}_{4}$ performed identically in producing plant height $(65.21-65.27 \mathrm{~cm})$, and number (7.16-7.26) and weight (413.3-430.0 g) of tuber/ hill which were significantly lower than $\mathrm{T}_{1}$.

Table 3. Yield and yield contributing characters of potato in farmer's field, Rangpur

\begin{tabular}{|c|c|c|c|c|c|}
\hline $\begin{array}{l}\text { Treatments } \\
\text { (Cropping Patterns) }\end{array}$ & $\begin{array}{l}\text { Plant height } \\
(\mathrm{cm})\end{array}$ & $\begin{array}{l}\text { Stem/hill } \\
\text { (no) }\end{array}$ & $\begin{array}{l}\text { Tuber/ hill } \\
\text { (no) }\end{array}$ & $\begin{array}{l}\text { Weight } \\
\text { Tuber/ hill } \\
\text { (g) }\end{array}$ & $\begin{array}{l}\text { Yield } \\
\left(\text { t ha-1) }^{-1}\right.\end{array}$ \\
\hline $\mathrm{T}_{1}=$ BINA7-Pot-Mung & $68.97 \mathrm{a}$ & 3.30 & $8.76 a$ & 466.7 a & $26.10 \mathrm{a}$ \\
\hline $\mathrm{T}_{2}=$ BR11-Pot-Fallow & $65.23 \mathrm{~b}$ & 3.30 & $7.26 \mathrm{~b}$ & $430.0 \mathrm{~b}$ & $24.79 \mathrm{~b}$ \\
\hline $\mathrm{T}_{3}=\mathrm{BINA7}-\mathrm{P}+\mathrm{M}-\mathrm{Mung}$ & $65.21 \mathrm{~b}$ & 3.33 & $7.20 \mathrm{~b}$ & $413.3 \mathrm{~b}$ & $23.65 b$ \\
\hline $\mathrm{T}_{4}=\mathrm{BR} 11-\mathrm{Pot} / \mathrm{Maize}$ & $65.27 \mathrm{~b}$ & 3.30 & $7.16 b$ & $423.3 \mathrm{~b}$ & $23.61 \mathrm{~b}$ \\
\hline $\mathrm{CV}(\%)$ & 2.71 & 4.7 & 8.67 & 4.2 & 3.21 \\
\hline Level of Significance & $*$ & NS & $*$ & $*$ & $*$ \\
\hline LSD (0.05) & 3.58 & 0.31 & 1.32 & 36.36 & 1.57 \\
\hline
\end{tabular}

*Significant at 0.05 level of significance

Letters in a column having similar or no letter did not differ significantly

\section{Maize}

Intercropped $\left(T_{3}\right)$ and Relay $\left(T_{4}\right)$ maize did not vary significantly in the parameters studied (Table 4$)$.

Table 4. Yield and yield contributing characters of maize in farmer's field, Rangpur

\begin{tabular}{llllllll}
\hline $\begin{array}{l}\text { Treatments } \\
\text { (Cropping }\end{array}$ & $\begin{array}{l}\text { Plant } \\
\text { population } \\
/ \mathrm{m}^{2}(\mathrm{no})\end{array}$ & $\begin{array}{l}\text { Plant } \\
\text { height } \\
(\mathrm{cm})\end{array}$ & $\begin{array}{l}\text { Cobs/ } \\
\text { plant } \\
(\mathrm{no})\end{array}$ & $\begin{array}{l}\text { Cob } \\
\text { length } \\
(\mathrm{cm})\end{array}$ & $\begin{array}{l}\text { Grains/ } \\
\text { cob } \\
(\mathrm{no})\end{array}$ & $\begin{array}{l}1000 \\
\text { grain } \\
\text { weight } \\
(\mathrm{g})\end{array}$ & $\begin{array}{l}\text { Yield } \\
\left(\mathrm{tha}^{-1}\right)\end{array}$ \\
\hline $\mathrm{T}_{1}=$ BINA7-Pot-Mung & - & - & - & - & - & - & - \\
$\mathrm{T}_{2}=$ BR11-Pot-Fallow & - & - & - & - & - & - & - \\
$\mathrm{T}_{3}=$ BINA7-P+M-Mung & 5.33 & 226.33 & 1.03 & 16.97 & 513.33 & 28.5 & 8.21 \\
$\mathrm{~T}_{4}=$ BR11-Pot/Maize & 5.27 & 230.67 & 1.13 & 17.3 & 520 & 28.57 & 7.92 \\
\hline CV $(\%)$ & 4.32 & 5.59 & 6.5 & 6.05 & 4.32 & 3.39 & 3.02 \\
\hline Level of Significance & $\mathrm{NS}$ & $\mathrm{NS}$ & $\mathrm{NS}$ & $\mathrm{NS}$ & $\mathrm{NS}$ & $\mathrm{NS}$ & $\mathrm{NS}$ \\
\hline LSD & 0.80 & 44.88 & 0.24 & 3.64 & 78.42 & 3.99 & 0.85 \\
\hline
\end{tabular}

*Significant at 0.05 level of significance

Letters in a column having similar or no letter did not differ significantly 


\section{Mungbean}

Number of pods/plant and yield showed significant variation due to treatment (Table 5). Mungbean after sole potato $\left(\mathrm{T}_{1}\right)$ gave higher number of pods/plant (17.25), and yield (1.47 t ha $^{-1}$ ) which is significantly higher than those of $\mathrm{T}_{3}$ (14.89 and $1.28 \mathrm{t} \mathrm{ha}^{-1}$, respectively). Higher number of pods/plant contributed to higher yield in $\mathrm{T}_{1}$.

Table 5. Yield and yield contributing characters of mungbean in farmer's field, Rangpur

\begin{tabular}{lllllll}
\hline $\begin{array}{l}\text { Treatments } \\
\text { (Croppingpatterns) }\end{array}$ & $\begin{array}{l}\text { Plant height } \\
(\mathrm{cm})\end{array}$ & $\begin{array}{l}\text { Pods/ } \\
\text { plant }(\mathrm{no})\end{array}$ & $\begin{array}{l}\text { Pod length } \\
(\mathrm{cm})\end{array}$ & $\begin{array}{l}\text { Seeds/pod } \\
(\mathrm{no})\end{array}$ & $\begin{array}{l}\text { 1000 seed } \\
\text { wt }(\mathrm{g})\end{array}$ & $\begin{array}{l}\text { Yield } \\
\left(\mathrm{t} \mathrm{ha}^{-1}\right)\end{array}$ \\
\hline $\mathrm{T}_{1}=$ BINA7-Pot-Mung & 53.3 & $17.25 \mathrm{a}$ & 7.03 & 7.32 & 41.90 & $1.47 \mathrm{a}$ \\
$\mathrm{T}_{2}=$ BR11-Pot-Fallow & - & - & - & - & - & - \\
$\mathrm{T}_{3}=$ BINA7-P+M-Mung & 55.07 & $14.89 \mathrm{~b}$ & 7.01 & 6.65 & 40.67 & $1.28 \mathrm{~b}$ \\
$\mathrm{~T}_{4}=$ BR11-Pot/Maize & - & - & - & - & - & - \\
\hline CV $(\%)$ & 3.63 & 3.61 & 5.8 & 3.55 & 2.08 & 3.19 \\
\hline Level of Significance & NS & $*$ & NS & NS & NS & $*$ \\
\hline LSD & 6.91 & 2.03 & 1.43 & 0.87 & 3.01 & 0.15 \\
\hline
\end{tabular}

*Significant at 0.05 level of significance

Letters in a column having similar or no letter did not differ significantly

\section{Economic Analysis}

\section{Economic analy sis of $T$. aman rice}

Closer or similar gross return (GR), gross margin (GM) and benefit cost return (BCR) was noticed among the treatments indicating that BR11 and
BINA dhan 7 offers more or less equal monetary benefit (Table 6). GR ranged from Tk. 63360 to 66400, GM from Tk. 30125 to 32000 and BCR from 1.91 to 1.93 .

Table 6. Economic analysis of T. aman rice in farmer's field, Rangpur

\begin{tabular}{llllll}
\hline $\begin{array}{l}\text { Treatments } \\
\text { (Cropping patterns) }\end{array}$ & $\begin{array}{l}\text { Yield } \\
\left(\text { t ha }^{-1}\right)\end{array}$ & $\begin{array}{l}\text { Gross return } \\
\left(\text { Tk ha }^{-1}\right)\end{array}$ & $\begin{array}{l}\text { Production } \\
\text { cost }\left(\text { Tk ha }^{-1}\right)\end{array}$ & $\begin{array}{l}\text { Gross margin } \\
\left(\text { Tk ha }^{-1}\right)\end{array}$ & BCR \\
\hline $\mathrm{T}_{1}=$ BINA7-Pot-Mung & 3.98 & 63680 & 33235 & 30445 & 1.92 \\
$\mathrm{~T}_{2}=$ BR11-Pot-Fallow & 4.16 & 66560 & 34560 & 32000 & 1.93 \\
$\mathrm{~T}_{3}=$ BINA7-P+M-Mung & 3.96 & 63360 & 33235 & 30125 & 1.91 \\
$\mathrm{~T}_{4}=$ BR11-Pot/Maize & 4.15 & 66400 & 34560 & 31840 & 1.92 \\
\hline
\end{tabular}

Selling Price $=$ Tk. 16 per $\mathrm{kg}$

\section{Economic analysis of potato}

Highest GR (Tk. 235080), GM (Tk. 129760) and BCR (2.23) were calculated in improved pattern $\mathrm{T}_{1}$ and the lowest of those (Tk. 212490, Tk.
106620 and 2.01) was found in the farmers pattern $\mathrm{T}_{4}$ (Table 7). Potato in improved pattern $\left(T_{1}, T_{3}\right)$ showed better performance than farmers pattern $\left(T_{1}, T_{4}\right)$.

Table 7. Economic analysis of potato in farmer's field, Rangpur

\begin{tabular}{llllll}
\hline $\begin{array}{l}\text { Treatments } \\
\text { (Cropping patterns) }\end{array}$ & $\begin{array}{l}\text { Yield } \\
\left(\mathrm{t} \mathrm{ha}^{-1}\right)\end{array}$ & $\begin{array}{l}\text { Gross return } \\
\left(\text { Tk ha }^{-1}\right)\end{array}$ & $\begin{array}{l}\text { Production } \\
\text { cost }^{\left(T_{k} \mathrm{~h}^{-1}\right)} \text { ) }\end{array}$ & $\begin{array}{l}\text { Gross margin } \\
\left(\text { Tk ha }^{-1}\right)\end{array}$ & BCR \\
\hline $\mathrm{T}_{1}=$ BINA7-Pot-Mung & 26.12 & 235080 & 105320 & 129760 & 2.23 \\
$\mathrm{~T}_{2}=$ BR11-Pot-Fallow & 24.79 & 223110 & 111460 & 111650 & 2.00 \\
$\mathrm{~T}_{3}=$ BINA7-P+M-Mung & 23.65 & 212850 & 100320 & 112530 & 2.12 \\
$\mathrm{~T}_{4}=$ BR11-Pot/Maize & 23.61 & 212490 & 105870 & 106620 & 2.01 \\
\hline
\end{tabular}

Selling Price $=$ Tk. 09 per $\mathrm{kg}$

\section{Economic analysis of maize}

Higher GR (Tk. 90310), GM (Tk. 49935) and BCR (2.24) were calculated in improved pattern $T_{3}$ than those (Tk. 87120, Tk. 44525 and 2.05) of the farmers pattern $\mathrm{T}_{4}$ (Table 8 ). 
Table 8. Economic analysis of maize in farmer's field, Rangpur

\begin{tabular}{llllll}
\hline $\begin{array}{l}\text { Treatments } \\
\text { (Cropping patterns) }\end{array}$ & $\begin{array}{l}\text { Yield } \\
\left(\text { t ha }^{-1}\right)\end{array}$ & $\begin{array}{l}\text { Gross return } \\
\left.\text { (Tk ha }^{-1}\right)\end{array}$ & $\begin{array}{l}\text { Production } \\
\text { cost }(\text { Tk ha-1) }\end{array}$ & $\begin{array}{l}\text { Gross margin } \\
\text { (Tk ha-1) }\end{array}$ & BCR \\
\hline $\mathrm{T}_{1}=$ BINA7-Pot-Mung & - & - & - & - & - \\
$\mathrm{T}_{2}=$ BR11-Pot-Fallow & - & - & - & - & - \\
$\mathrm{T}_{3}=$ BINA7-P+M-Mung & 8.21 & 90310 & 40375 & 49935 & 2.24 \\
$\mathrm{~T}_{4}=$ BR11-Pot/Maize & 7.92 & 87120 & 42595 & 44525 & 2.05 \\
\hline
\end{tabular}

Selling Price $=$ Tk. 11 per $\mathrm{kg}$

\section{Economic analysis of mungbean}

Mungbean after sole potato $\left(\mathrm{T}_{1}\right)$ showed higher GR (Tk. 58800), GM (Tk. 35230) and BCR (2.49) than those (Tk. 51200, Tk. 27630 and 2.17) of $\mathrm{T}_{3}$ (Table 9).

Table 9. Economic analysis of Mungbean in farmer's field, Rangpur

\begin{tabular}{llllll}
$\begin{array}{l}\text { Treatments } \\
\text { (Cropping Patterns) }\end{array}$ & $\begin{array}{l}\text { Yield } \\
\left(\text { t ha }^{-1}\right)\end{array}$ & $\begin{array}{l}\text { Gross return } \\
\left(\text { Tk ha }^{-1}\right)\end{array}$ & $\begin{array}{l}\text { Production } \\
\text { cost }\left(\text { Tk ha }^{-1}\right)\end{array}$ & $\begin{array}{l}\text { Gross margin } \\
\left(\text { Tk ha }^{-1}\right)\end{array}$ & BCR \\
\hline $\mathrm{T}_{1}=$ BINA7-Pot-Mung & 1.47 & 58800 & 23570 & 35230 & 2.49 \\
$\mathrm{~T}_{2}=$ BR11-Pot-Fallow & - & - & - & - & - \\
$\mathrm{T}_{3}=$ BINA7-P+M-Mung & 1.28 & 51200 & 23570 & 27630 & 2.17 \\
$\mathrm{~T}_{4}=$ BR11-Pot/Maize & - & - & - & - & -
\end{tabular}

Selling Price $=$ Tk. 40 per kg

\section{Economic analysis of whole pattern}

Highest GR (Tk. 417720) and GM (Tk. 220220) were calculated in improved pattern $\mathrm{T}_{3}$ and the lowest of those (Tk. 289670, Tk. 146020) in farmers pattern $\mathrm{T}_{2}$ (Table 10) The other improved pattern $\mathrm{T}_{3}$ was the second highest performer considering GR and GM. But BCR (2.21) was highest in $\mathrm{T}_{1}$ and second highest in $\mathrm{T}_{3}$. The result indicated that the improved patterns $\left(T_{1}, T_{3}\right)$ were better than farmers pattern $\left(T_{2}, T_{4}\right)$.

Table 10. Economic analysis of the whole patterns in farmer's field Rangpur

\begin{tabular}{lllll}
\hline $\begin{array}{l}\text { Treatments } \\
\text { (Cropping patterns) }\end{array}$ & $\begin{array}{l}\text { Gross return } \\
\left(\text { Tk ha }^{-1}\right)\end{array}$ & $\begin{array}{l}\text { Production cost } \\
\left(\text { Tk ha }^{-1}\right)\end{array}$ & $\begin{array}{l}\text { Gross margin } \\
\left(\text { Tk ha }^{-1}\right)\end{array}$ & BCR \\
\hline $\mathrm{T}_{1}=$ BINA7-Pot-Mung & 357560 & 162125 & 195435 & 2.21 \\
$\mathrm{~T}_{2}=$ BR11-Pot-Fallow & 289670 & 146020 & 143650 & 1.98 \\
$\mathrm{~T}_{3}=$ BINA7-P+M-Mung & 417720 & 197500 & 220220 & 2.12 \\
$\mathrm{~T}_{4}=$ BR11-Pot/Maize & 365850 & 183025 & 182825 & 2.00 \\
\hline
\end{tabular}

Return of improved patterns over the $\left(T_{2}\right)$ (Table 11). Similarly, the other improved farmers patterns

The improved pattern $\left(\mathrm{T}_{1}\right)$ gave GR Tk. 67890 pattern $\left(\mathrm{T}_{3}\right)$ showed Tk. 51870 and Tk. 37395 higher than farmers pattern $\left(\mathrm{T}_{4}\right)$. and GM Tk. 51785 higher than farmers pattern

Table 11. Return of improved patterns over the farmers patterns in farmer's field, Rangpur

\begin{tabular}{lllll}
\hline $\begin{array}{l}\text { Treatments } \\
\text { (Cropping patterns) }\end{array}$ & $\begin{array}{l}\text { Gross return } \\
\left(\text { Tk ha }^{-1}\right)\end{array}$ & $\begin{array}{l}\text { Production } \\
\text { cost (Tk ha-1) }\end{array}$ & $\begin{array}{l}\text { Gross margin } \\
\left.\text { (Tk ha }^{-1}\right)\end{array}$ & BCR \\
\hline $\begin{array}{l}\mathrm{T}_{1} \text { over T } \\
\text { (BINA7-Pot-Mung over BR11-Pot-Fallow) }\end{array}$ & 67890 & 16105 & 51785 & 4.22 \\
$\begin{array}{l}\mathrm{T}_{3} \text { Over T } \\
\text { (BINA7-P+M-Mung over BR11-Pot/Maize) }\end{array}$ & 51870 & 14475 & 37395 & 3.58 \\
\hline
\end{tabular}

\section{Job Creation}

The improved pattern $\mathrm{T}_{1}$ and $\mathrm{T}_{3}$ created 45 working day ( 1 day $=8$ hours work) job for a labour for harvesting early matured rice in the Monga/ jobless period (October) while farmers' pattern gives only 3 days work (Table 12). Again, $\mathrm{T}_{3}$ gave total 440 days work throughout the year which was the highest, followed by $\mathrm{T}_{4}$ (392 days), $\mathrm{T}_{1}$ (372 days) and the lowest in $\mathrm{T}_{2}$ (305 days). It indicates that the improved patterns created higher job opportunity than the farmers' pattern. 
Table 12. Labour distribution in monga period (day ha-1)

\begin{tabular}{lcl}
\hline Treatments & $\begin{array}{c}\text { Labour use }\left(\text { day ha }^{-1} \text { ) in monga period }\right. \\
\text { (October) }\end{array}$ & Total labour \\
\hline $\mathrm{T}_{1}=$ BINA7-Pot-Mung & 45 & $108+189+75=372$ \\
$\mathrm{~T}_{2}=$ BR11-Pot-Fallow & 3 & $108+197+0=305$ \\
$\mathrm{~T}_{3}=$ BINA7-P+M-Mung & 45 & $108+266+75=440$ \\
$\mathrm{~T}_{4}=$ BR11-Pot/Maize & 3 & $108+197+80=392$ \\
\hline
\end{tabular}

\section{Conclusion}

The result showed that mungbean could successfully be accommodated in potato based cropping pattern. Intercropping potato + maize, rather than relay cropping potato with maize not only increase total productivity, but also facilitate two and half month time to grow mungbean before T. aman cultivation. The Improved (mungbean included) patterns increased total production and monetary return. Short duration T. Aman rice created job opportunity for the poor labour in Monga/ off period (October). Pulse production increased. The improved pattern $\mathrm{T}_{1}=$ BINA dhan 7-Potato-Mungbean can be suggested against farmers pattern $\mathrm{T}_{2}=$ BR11 - Potato Fallow and the improved pattern $\mathrm{T}_{3}=$ BINA dhan 7 - Potato + Maize intercropping - Mungbean can be suggested against farmers pattern $\mathrm{T}_{4}=$ BR11-Pot/Maize. The mungbean included improved cropping patterns can be suggested for increased production, economic return and Monga mitigation (work opportunity in off period) in Rangpur.

\section{References}

BARC. 2001. Impact of land degradation of Bangladesh: A changing scenario in agriciltural land use. BARC, Farmgate, Dhaka. pp. 39-102.
Chowdhury, D.A., Shafiqul Islam, M. and Muktadir Alam, M. 2003. Integrated nutrient management for potato- T. aus-T. Aman cropping system. OFRD, BARI, Gazipur and SFFP (Integrated Soil Fertility and Fertilizer Management Project), DAE, Farmgate, Dhaka. pp. 1-5.

Dey, T.K. 2001. Occurrence and management of bacterial wilt of potato and survivability of Talstonia soloni. Ph.D. Thesis. Dept. Pl. Path. Bangabandhu Sheikh Mujibur Rahman Agricultural University, Salna, Gazipur. P.185.

Gomez, K.A. and Gomez, A.A. 1984. Statistical Procedures for Agricultural Research. John Wiley and sons, New York. p. 28.

Krishna, A. and Raikhelkar, S.V. 1997. Crop complementary in maize (Zea mays) when intercropped with different legumes. Indian J. Agric. Sci. 67: 291-294.

Luis, A. and Manrique. 1996. The potato in multiple cropping systems. J. Plant Nutr. 19 (10): 215-243.

Trenbath, B.R. 1993. Intercropping for the management of pests and diseases. Field Crops Res. 34 (3-4): 381-405.

Umrani, N.K., Sinadi and Dhonde, P.M. 1984. Studies on intercropping of pulses in sorghum. Indian J. Agric. Sci. 29 (1): 27-30. 